\title{
High Precision Vehicle Location Technologies Based on Traffic Lights
}

\author{
Yan Cao', Tao Lei ${ }^{2}$ \\ 1,2. School of Electronic and Information Engineering , Lanzhou Jiao tong University, Lanzhou,730070, China
}

\begin{abstract}
According to the low positioning precision and high cost of traditionall vehicle
Positioning technologies, and combined advantages of the newly arisen visible light communication

like no extra transmitter needed, no electromagnetic interference and free license, this paper proposed a vehiclepositioning method based on traffic light, which could generate the vehicle's position with the time Difference of arrival of the signal light. Also its improved methods based on plan revolutiontheory were discussed to overcome the deficientin non-coplanar condition and improve the positioning precision. Simulation results showed that these methods, with simple computation and low implementation cost, could realize a real-timehigh-precision positioning performance, and could meet the requirements of the intelligence transportation system.
\end{abstract}

KEYWORDS

\author{
Vehicle positioning \\ Traffic light \\ Message gateway \\ TDOA
}

Visible light communication

\section{INTRODUCTION}

High precision, high reliability and vehicle location information provided by Intelligent transportation systems (Intelligence Transportation System, ITS) is a basic condition for vehicle location and navigation. It is also the foundation to achieve traffic diversion, urban traffic intelligent scheduling, motor control and other uses [1-2]. Due to the usual building block for positioning signals, the existed satellite positioning systems which is most extensive and most widely used could not be used well in highrise cities with tunnel, underground car park and other closed areas. And civilian business positioning accuracy is of less than 10 meters, which has been unable to meet ITS increasing accuracy requirements of the system [3]. Existing wireless location technology mainly uses infrared [4], electromagnetic waves [5] [8], magnetic field [9], sound wave [10],ultrasonic wave [11] and other forms to send positioning signal. And also could realize high precision of wireless positioning [13] through realtime image information [14]. But these positioning technology need to install additional signal launch device, increasing the complexity of the system and the implementation cost. Radio frequency identification technology [5], wlan positioning

Copyright $(92016$ Yan Cao and Tao Lei doi: $10.18686 /$ esta.v3i1.3

This is an open-access article distributed under the terms of the Creative Commons Attribution Unported License (http://creativecommons.org/licenses/by-nc/4.0/), which permits unrestricted use, distribution, and reproduction in any medium, provided the original work is properly cited. technique [8], wireless sensor network location technology [6, 13] and uwb positioning technology [7] based on electromagnetic wave signal will also occupy a certain communication bandwidth, reducing the bandwidth of the communication system efficiency. And due to electromagnetic interference effect, it could not be applied to hospitals, airports and other environment where the RF signal was strictly limited. Positioning technology based on real-time image information necessary to locate the environment to build a huge database of images with poor real-time performance. To overcome the lack of the above positioning algorithm, combined advantages of the newly arisen visible light communication like no extra transmitter needed, no electromagnetic interference and free license [14], this paper proposes a traffic light based wireless location technology and improved techniques for non-coplanar problem, which can achieve real-time positioning with high accuracy to meet the ITS system for vehicle location requirements.

\section{Introduction}

nolHigh precision, high reliability and vehicle location information provided by Intelligent transportation systems (Intelligence Transportation System, ITS) is a basic condition for vehicle location and navigation. It is also the foundation to achieve traffic diversion, urban traffic intelligent scheduling, motor control and other uses [1-2] Due to the usual building block for positioning signals, the existed satellite positioning systems which is most extensive and most widely used could not be used well in high-rise cities with tunnel, underground car park and other closed areas. And civilian business positioning accuracy is of less than 10 meters, which has been 
unable to meet ITS increasing accuracy requirements of the system [3]. Existing wireless location technology mainly uses infrared [4], electromagnetic waves [5] [8], magnetic field [9], sound wave [10], ultrasonic wave [11] and other forms to send positioning signal. And also could realize high precision of wireless positioning [13] through real-time image information [14]. But these positioning technology need to install additional signal launch device, increasing the complexity of the system and the implementation cost. Radio frequency identification technology [5], wlan positioning technique [8], wireless sensor network location technology [6, 13] and uwb positioning technology [7] based on electromagnetic wave signal will also occupy a certain communication bandwidth, reducing the bandwidth of the communication system efficiency. And due to electromagnetic interference effect, it could not be applied to hospitals, airports and other environment where the RF signal was strictly limited. Positioning technology based on real-time image information necessary to locate the environment to build a huge database of images with poor real-time performance. To overcome the lack of the above positioning algorithm, combined advantages of the newly arisen visible light communication like no extra transmitter needed, no electromagnetic interference and free license [14], this paper proposes a traffic light based wireless location technology and improved techniques for non-coplanar problem, which can achieve real-time positioning with high accuracy to meet the ITS system for vehicle location requirements.

\section{Vehicle location technology based on the traffic light}

The vehicle positioning technology based on traffic lights is to use two optoelectronic receivers (Photodiode, PD) installed on the vehicle to receive the positioning signal from the traffic lights at the same time. According to the time difference of arrival (TDOA) between the two PD, compute the location relationship between the target vehicle (defined as the geometric center of two PD receivers) and the traffic lights. Finally, calculate the true position information of the target vehicle's location based on real information visible light communication technology acquired traffic lights (or on behalf of the position coordinate ID information). It can be divided into single lamp positioning technology and multi-lamp positioning technology according to the number of traffic lights.

\subsection{Single lamp positioning}

As shown in figure 1, F1 and F2 are two PD receivers symmetrically distributed on the $\mathrm{X}$ axis at the start moment, with a space of $2 \mathrm{C}$, moving along the positive direction of $\mathrm{Y}$ axis with a speed of $\mathrm{V} . F_{1}^{\prime}$ and $F_{2}^{\prime}$ are two PD receivers at $t$ moment, and T1 is the traffic light for sending positioning signal. According to the TDOA of visible light signal between the starting and $\mathrm{t}$ moment, $\Delta \mathrm{t} 1$ and $\Delta \mathrm{t} 2$, two hyperbolic curves with centrifugation rate of e1 and e2 are determined ( Respectively correspond to the above and below hyperbolic in figure 2, and the vertical dashed line is the left quasi line of the e1 hyperbolic curve).

$$
\begin{aligned}
& \sqrt{[x-(-c)]^{2}+y^{2}}=e_{1} \cdot\left[x-\left(-a_{1} / e_{1}\right)\right] \\
& \sqrt{[x-(-c)]^{2}+(y-\Delta y)^{2}}=e_{2} \cdot\left[x-\left(-a_{2} / e_{2}\right)\right]
\end{aligned}
$$

In which, $\Delta y=v \times t$ is the moving distance of receiver in $t$ interval time. The eccentricity $e=\frac{c}{a}, \mathrm{a}=\mathrm{C} \cdot \Delta \mathrm{t}$ is half the distance

difference of two hyperbolic focus from any point on the hyperbolic.

$\mathrm{C}=3 \times 108 \mathrm{~m} / \mathrm{s}$ is the velocity of propagation of visible light, and $\Delta$ $t$ is the time difference from Visible light signals to the two PD receivers. The four intersection points of two hyperbolas, t1, t2, t3 and $t 4$ are four solutions to the equations 1 and 2,

Because the travel time of visible light signal from detector $F 1$ is less than from detector $F 2$, the traffic lamp should is located within $x<0$ of left half plane, on the left half of hyperbolic curve, which can effectively remove the two fake solutions, $t 2$ and $t 4$; And non-full to receive of PD receiver decided traffic lamp should is located in receiver of maximum depending on field angle (Field of View, FoV) within, that $y>0$ and $y>\Delta y$, which can effectively remove the false solutions $\mathrm{t} 3$. It determines the location of traffic light $\mathrm{t} 1$ on the $\mathrm{XY}$ coordinate system coordinates, as well as the location relationship between traffic lights $t 1$ and $P D$ receiver. Combined with the real location information $(X, Y)$ of traffic lights $T 1$, final the real location

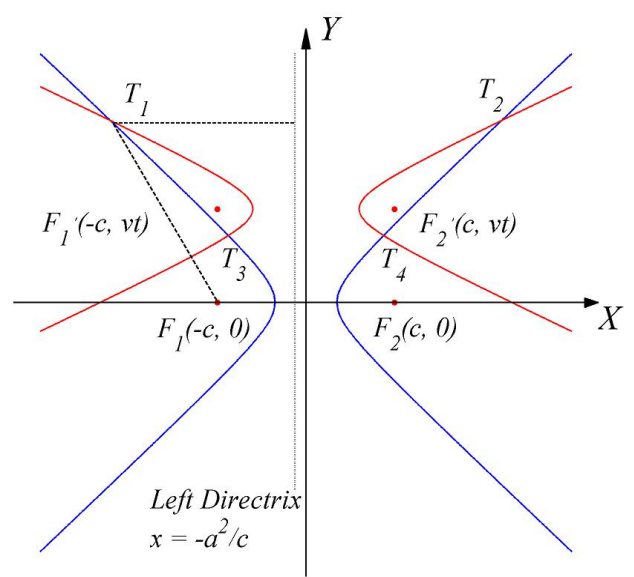

information $(X-X, Y-y)$ of target can be calculated (coordinate origin).

$$
\text { Figure } 1 \text { Single Lamp Positioning Technology }
$$

\subsection{Multi-lamp positioning}

When PD receiver can detect multiple transmit positioning signals from traffic light (at least two), you can determine a plurality of hyperbolic and finally calculate the real location information of the target according to the TDOA of visible signal between the two PD receivers.

As shown in figure2, F1 and F2 two PD receivers symmetrically distributed on the $\mathrm{X}$ axis, T1 and T2 are two different traffic lights emission positioning signal. According to the TDOA of visible signal at $\mathrm{T} 1$ and $\mathrm{T} 2$ between the two PD receivers, $\Delta \mathrm{t} 1$ and $\Delta \mathrm{t} 2$, determine eccentricity two hyperbolic with e1 and e2.

$$
\sqrt{\left[x_{1}-(-c)\right]^{2}+y_{1}^{2}}=e_{1} \cdot\left[x_{1}-\left(-a_{1} / e_{1}\right)\right]
$$

$$
\begin{aligned}
& \sqrt{\left[x_{2}-(-c)\right]^{2}+y_{2}^{2}}=e_{2} \cdot\left[x_{2}-\left(-a_{2} / e_{2}\right)\right] \\
& \sqrt{\left(x_{1}-x_{2}\right)^{2}+\left(y_{1}-y_{2}\right)^{2}}=\sqrt{\left(X_{1}-X_{2}\right)^{2}+\left(Y_{1}-Y_{2}\right)^{2}}
\end{aligned}
$$

$\frac{x_{1}-x_{2}}{X_{1}-X_{2}}=\frac{y_{1}-y_{2}}{Y_{1}-Y_{2}}$ 
In which, $(x 1, y 1)$ and $(x 2, y 2)$ are the location of traffic lights $T 1$ and $T 2$ in the $X Y$ coordinate system. $(X 1, Y 1)$ and $(X 2, Y 2)$ are the real location of T1 and T2. Similar to a single light positioning technology, due to the non-omni directional reception PD receiver restrictions, there is $\mathrm{y} 1>0, \mathrm{y} 2>0$; At the same time, if the TDOA of the visible light signal is $\Delta \mathrm{t} 1>0$ and $\Delta \mathrm{t} 2>0$, there is $\mathrm{x} 1>0, \mathrm{x} 2>0$; If the TDOA is $\Delta \mathrm{t} 1<0, \Delta \mathrm{t} 2<0$, there is $\mathrm{x} 1<0, \mathrm{x} 2<0$. Solve the above equation of false solutions and remove the fault solutions to get the position of traffic lights $\mathrm{T} 1$ and $\mathrm{T} 2$ on the $\mathrm{XY}$ coordinate system, (x1, $y 1)$ and ( $x 2, y 2)$. Combined with the real location information of traffic lights $\mathrm{T} 1(\mathrm{X} 1, \mathrm{Y} 1)$ and $\mathrm{T} 2(\mathrm{X} 2, \mathrm{Y} 2)$, calculate (coordinate origin) the real location information $(X 1-X 1, Y 1-Y 1)$ or $(X 2-X 2, Y 2-Y 2)$ of the target.

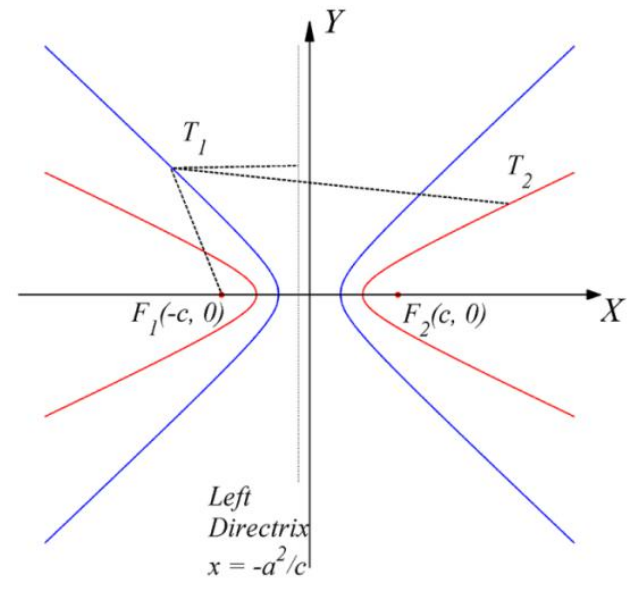

Figure 2 Multi-lamp positioning technologies

\section{Problems for non-coplanar positioning technology improvements}

Based on the above traffic light positioning technology is only applicable to the condition that traffic lights and the receivers are on the same plane. However as shown in Figure 3 and 4, in practical applications, traffic lights and receivers are often in different planes, the computer model to be modified. In this section the improvement of positioning technology under non-coplanar condition will be discussed.

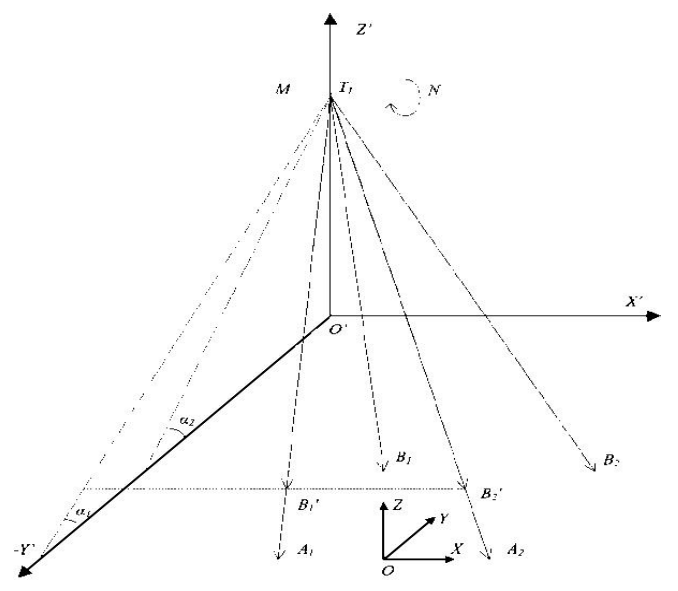

Figure. 3 single lamp positioned to improve technology

As shown in figure 3 , ways to improve the TDOA of single lamp positioning technology, $\mathrm{A} 1$ and $\mathrm{A} 2$ are two receivers $(-\mathrm{c}, 0,0)$ and $(\mathrm{c}$, $0,0)$ symmetrically distributed in the $X$-axis coordinate $X Y Z O$ in the starting time. To simplify the calculation, it is assumed that both receivers moves along the $Y$ axis in XOY plane with the speed of $v$, then the position of the two receivers at $\mathrm{t}$ are $\mathrm{B} 1(-\mathrm{c}, \Delta \mathrm{y}, 0)$ and $\mathrm{B} 2$ (c, $\Delta y, 0)$. The coordinates of traffic light $T 1$ on the $X Y Z O$ coordinate system is $(x, y, z)$, the angle between the T1A1A2 plane and the XOY plane is $\alpha_{1}=\arg \tan \left(\frac{z}{y}\right)$, and the angle between the T1B1B2 plane and the XOY plane is $\alpha_{2}=\arg \tan \left(\frac{z}{y-\Delta y}\right)$ Along with the intersection line $\mathrm{MN}$ between T1A1A2 plane and T1B1B2 plane, rotate the plane T1B1B2 with $\Delta \alpha=\alpha 2-\alpha 1$, then coincides with the plane T1A1A2, getting the mapping points $B_{1}^{\prime}$ and $B_{2}^{\prime}$ of receivers $\mathrm{B} 1$ and B2. According to the coordinate transformation theory, the mapping points $B_{1}^{\prime}$ and $B_{2}^{\prime}$ Coordinates on the coordinate system XYZO are $\left(x_{B 1}^{\prime}, y_{B 1}^{\prime}, z_{B 1}^{\prime}\right)$ and $\left(x_{B 2}^{\prime}, y_{B 2}^{\prime}, z_{B 2}^{\prime}\right)$
$\begin{aligned}\left(x_{B 1}^{\prime}, y_{B 1}^{\prime}, z_{B 1}^{\prime}, 1\right) & =\left(x_{B 1}, y_{B 1}, z_{B 1}, 1\right) \cdot R(\Delta \alpha) \\ & =(-c, \Delta y, 0,1) \cdot T \cdot R_{x}(\Delta \alpha) \cdot T^{-1} \\ \left(x_{B 2}^{\prime}, y_{B 2}^{\prime}, z_{B 2}^{\prime}, 1\right) & =\left(x_{B 2}, y_{B 2}, z_{B 2}, 1\right) \cdot R(\Delta \alpha) \\ & =(c, \Delta y, 0,1) \cdot T \cdot R_{x}(\Delta \alpha) \cdot T^{-1}\end{aligned}$

which $T=\left[\begin{array}{llll}1 & 0 & 0 & 0 \\ 0 & 1 & 0 & 0 \\ 0 & 0 & 1 & 0 \\ 0 & y & z & 1\end{array}\right]$ and $\quad R_{x}(\Delta \alpha)=\left[\begin{array}{cccc}1 & 0 & 0 & 0 \\ 0 & \cos (\Delta \alpha) & \sin (\Delta \alpha) & 0 \\ 0 & -\sin (\Delta \alpha) & \cos (\Delta \alpha) & 0 \\ 0 & 0 & 0 & 1\end{array}\right]$ are respectively transformation matrix and rotation matrix, $T^{-1}=\left[\begin{array}{cccc}1 & 0 & 0 & 0 \\ 0 & 1 & 0 & 0 \\ 0 & 0 & 1 & 0 \\ 0 & -y & -z & 1\end{array}\right]$ the distance difference between the traffic lights and the two receivers' mapping point ${ }^{\prime}$ and $B_{2}^{\prime}$

$$
\begin{aligned}
a_{2}^{\prime}= & \frac{1}{2}\left[\sqrt{\left(x-x_{B 1}^{\prime}\right)^{2}+\left(y-y_{B 1}^{\prime}\right)^{2}+\left(z-z_{B 1}^{\prime}\right)^{2}}\right. \\
& \left.-\sqrt{\left(x-x_{B 2}^{\prime}\right)^{2}+\left(y-y_{B 2}^{\prime}\right)^{2}+\left(z-z_{B 2}^{\prime}\right)^{2}}\right]
\end{aligned}
$$

Rewrite the formula (1) and (2)

$$
\begin{aligned}
& \sqrt{[x-(-c)]^{2}+y^{2}+z^{2}}=e_{1} \cdot\left[x-\left(-a_{1} / e_{1}\right)\right] \\
& \sqrt{\left(x-x_{B 1}^{\prime}\right)^{2}+\left(y-y_{B 1}^{\prime}\right)^{2}+\left(z-z_{B 1}^{\prime}\right)^{2}} \\
& =e_{2}^{\prime} \cdot\left[x-\left(-a_{2}^{\prime} / e_{2}^{\prime}\right)\right]
\end{aligned}
$$


In which, $\quad e_{2}^{\prime}=\frac{c}{a_{2}^{\prime}}$ is the eccentricity of the hyperbolic determined by traffic lights T1And two receivers mapping point ${ }^{\prime}{ }_{1}^{\prime}$ and $B_{2}^{\prime}$. Solve the above equations and use similar restrictions of single lamp coplanar positioning technology, the position coordinates $(x, y, z)$ of traffic light $\mathrm{T} 1$ on $\mathrm{XYZO}$ coordinate can be calculated. Combined with the true position $(X, Y, Z)$ of traffic lights $T$ 1, finally obtain the actual position $(X-x, Y-y, Z-z)$ of conversion target (XYZO coordinate origin).

As shown in figure 4 , ways to improve the TDOA of single lamp positioning technology, $\mathrm{A} 1$ and $\mathrm{A} 2$ are two receivers $(-\mathrm{c}, 0,0)$ and $(\mathrm{c}$, $0,0)$ symmetrically distributed in the $X$-axis coordinate $X Y Z O$. The coordinates of LED signal light T1 and T2 on the coordinate system $\mathrm{XYZO}$ are $(\mathrm{x} 1, \mathrm{y} 1, \mathrm{~h} 1)$ and $(\mathrm{x} 2, \mathrm{y} 2, \mathrm{~h} 2)$. Similar to the ways to improve the single lamp positioning technology, rotate the plane $\mathrm{T} 2 \mathrm{~A} 1 \mathrm{~A} 2$, then coincides with the plane $\mathrm{T} 1 \mathrm{~A} 1 \mathrm{~A} 2$, getting the coordinates $\left(x_{2}^{\prime}, y_{2}^{\prime}, h_{2}^{\prime}\right)$ and the real coordinates $\left(X_{2}^{\prime}, Y_{2}^{\prime}, H_{2}^{\prime}\right)$ of mapping point $T_{2}^{\prime}$ of the LED signal light T2 coordinates on the coordinate system XYZO.

$$
\begin{aligned}
& \left(x_{2}^{\prime}, y_{2}^{\prime}, h_{2}^{\prime}, 1\right)=\left(x_{2}, y_{2}, h_{2}, 1\right) \cdot R_{x}(\Delta \alpha) \\
& \left(X_{2}^{\prime}, Y_{2}^{\prime}, H_{2}^{\prime}\right)=\left(X_{2}, Y_{2}, H_{2}\right)-\left(x_{2}-x_{2}^{\prime}, y_{2}-y_{2}^{\prime}, h_{2}-h_{2}^{\prime}\right)
\end{aligned}
$$

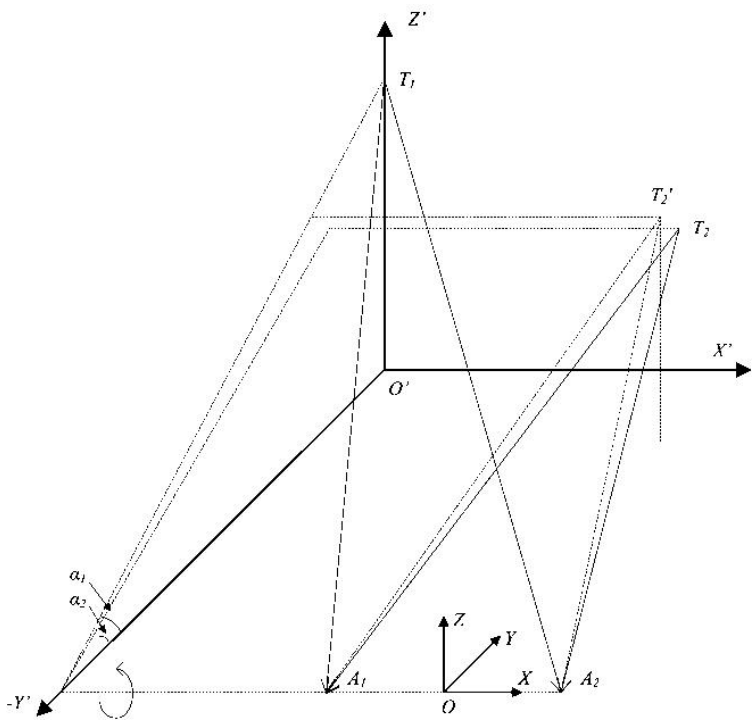

4 Multi-lamp technology to improve positioning

The distance difference between mapping point $T_{2}^{\prime}$ of the traffic light T2 and the two receivers

$$
\begin{aligned}
a_{2}^{\prime} & =\frac{1}{2}\left\{\sqrt{\left[x_{2}^{\prime}-(-c)\right]^{2}+\left(y^{\prime}\right)_{2}^{2}+\left(h_{2}^{\prime}\right)^{2}}\right. \\
& \left.-\sqrt{\left(x_{2}^{\prime}-c\right)^{2}+\left(y^{\prime}\right)_{2}^{2}+\left(h_{2}^{\prime}\right)^{2}}\right\}
\end{aligned}
$$

Rewrite the equation (3) to (6)

$$
\begin{aligned}
& \sqrt{\left[x_{1}-(-c)\right]^{2}+y_{1}^{2}+h_{1}^{2}}=e_{1} \cdot\left[x_{1}-\left(-a_{1} / e_{1}\right)\right] \\
& \sqrt{\left[x_{2}^{\prime}-(-c)\right]^{2}+\left(y_{2}^{\prime}\right)^{2}+\left(h_{2}^{\prime}\right)^{2}}=e_{2}^{\prime} \cdot\left[x_{2}^{\prime}-\left(-a_{2}^{\prime} / e_{2}^{\prime}\right)\right]
\end{aligned}
$$

$$
\begin{aligned}
& \sqrt{\left(x_{1}-x_{2}^{\prime}\right)^{2}+\left(y_{1}-y_{2}^{\prime}\right)^{2}+\left(h_{1}-h_{2}^{\prime}\right)^{2}} \\
& =\sqrt{\left(X_{1}-X_{2}^{\prime}\right)^{2}+\left(Y_{1}-Y_{2}^{\prime}\right)^{2}+\left(H_{1}-H_{2}^{\prime}\right)^{2}} \\
& \frac{x_{1}-x_{2}^{\prime}}{X_{1}-X_{2}^{\prime}}=\frac{y_{1}-y_{2}^{\prime}}{Y_{1}-Y_{2}^{\prime}}=\frac{h_{1}-h_{2}^{\prime}}{H_{1}-H_{2}^{\prime}}
\end{aligned}
$$

In which, $e_{2}^{\prime}=\frac{c}{a_{2}^{\prime}}$ is the eccentricity of the hyperbolic determined by mapping point $T_{2}^{\prime}$ of traffic lights T2 and the two receivers. Solve the above equations and use similar restrictions of multi lamp coplanar positioning technology, the position coordinates $(x 1, y 1, h 1)$ and $\left(x_{2}^{\prime}, y_{2}^{\prime}, h_{2}^{\prime}\right)$ of mapping point $T_{2}^{\prime}$ of traffic lights T2 and traffic light T1 on $\mathrm{XYZO}$ coordinate can be calculated. Combined with the true position $\left(X_{1}, Y_{1}, H_{1}\right)$ and $\left(X_{2}^{\prime}, Y_{2}^{\prime}, H_{2}^{\prime}\right)$ of traffic lights, finally obtain the conversion of the receiver's position $(X 1-X 1, Y 1-Y 1, H 1-H 1)$ or $\left(X_{2}^{\prime}-x^{\prime}, Y_{2}^{\prime}-y_{2}^{\prime}, H_{2}^{\prime}-h_{2}^{\prime}\right)$.

\section{Simulation and Analysis}

Positioning error is defined positioning technology [15]

$$
\operatorname{Bias}=\sqrt{\operatorname{Bias}_{X}^{2}+\operatorname{Bias}_{Y}^{2}}
$$

In which, Bias $X$ and Bias $Y$ respectively are positioning error of positioning technology in the $\mathrm{X}$-axis and $\mathrm{Y}$-axis. Without taking wrong place of traffic light and the TDOA of visible light signal between the two receivers into account, as shown in figure 5 , set the traffic lights T1 and T2 as two receivers located on the left and right of the center of $3 \mathrm{~m}$ at $1 \mathrm{~m}$, the receiver move along the $Y$ axis opposite the traffic light.
Evaluate

True height of traffic light $T_{1}, H_{1} \quad 6 \mathrm{~m}$

True height of traffic light $T_{2}, H_{2} \quad 4 \mathrm{~m}$

True height of the receiver, $h \quad 1 \mathrm{~m}$

Space between two receivers, $2 c \quad 1 m$

Receiver measurement time $0.1 \mathrm{~s}$ interval, $t$ 
Table 1 shows the system parameters, figure 6 gives a comparison of receiver velocity for the next $10 \mathrm{mps}$ and $30 \mathrm{mps}$ case, and shows the error curve TDOA single lamp positioning technology caused by non-coplanar, only based on a visible light signal launched by traffic light T1. As can be seen, when the distance between the receiver and the traffic light is reduced, the error of positioning unused rotational improvements will increase slowly and then rapidly increases; and when the greater the velocity, the mobile receiver measurement time interval distance $\Delta y$ the larger, the greater the positioning error.

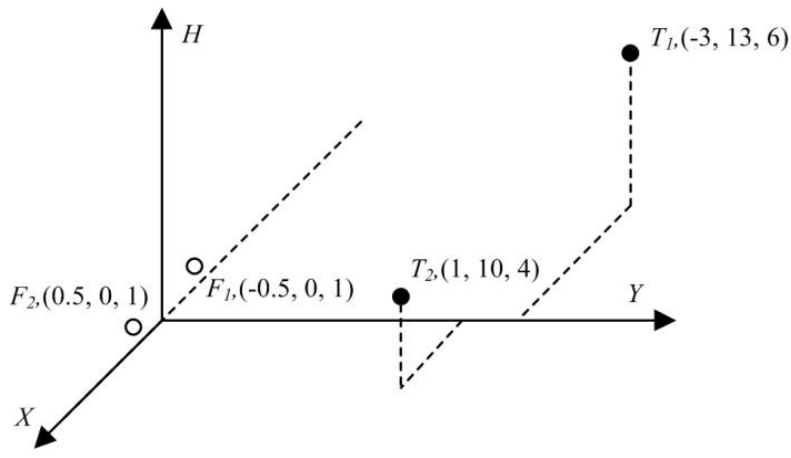

5 Single light positioning technology positioning error

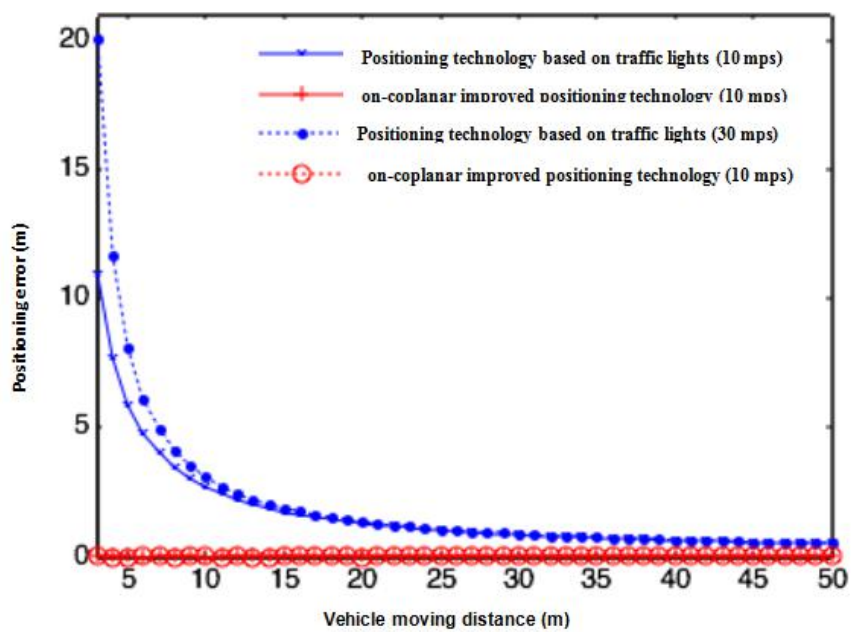

Figure. 6 Single light positioning technology positioning error

Figure 7 gives a comparison of receiver velocity for the next $10 \mathrm{mps}$ and $30 \mathrm{mps}$ case, and shows the error curve TDOA single lamp positioning technology caused by non-coplanar. Similarly, when the distance between the receiver and the traffic light is reduced, the error of positioning unused rotational improvements will increase slowly and then rapidly increases; Compared with TDOA single lamp positioning technology, multi-lamp TDOA positioning technology is with better positioning accuracy of performance. When the distance between the receiver and traffic lights is $5 \mathrm{~m}$, the error of multi-lamp positioning technology unused rotational improvements is only $3.4 \mathrm{~m}$, which corresponds to the receiver with velocity of $10 \mathrm{mps}$ and 30 $\mathrm{mps}$, the error of positioning unused rotational improvements of TDOA single lamp is $5.9 \mathrm{~m}$ and $8.1 \mathrm{~m}$. Here only study analyzed the multi-positioning technology based on two traffic lights lamp. TDOA

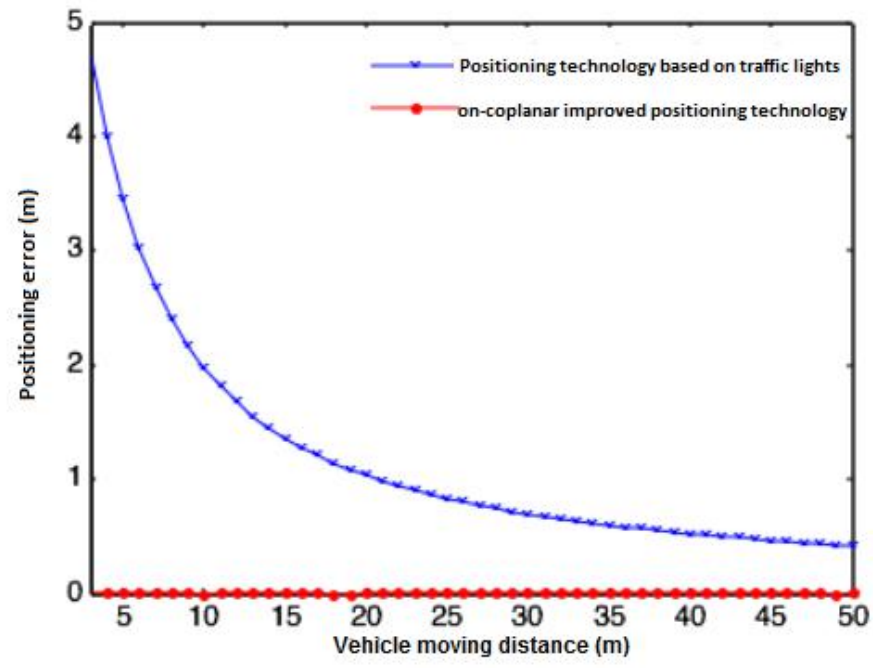

7 Multi-lamp positioning technology positioning error

\section{Concluding Remarks}

This paper presents a novel technique based vehicle positioning of traffic lights, and improved techniques for non-coplanar problem of traffic lights and the receiverPD. Simulation results show that the positioning technology can achieve higher accuracy real-time positioning, intelligent transportation system to meet the requirements of the vehicle location.

\section{References}

1. JIANG Xin-hua, Chen Yu, Zhu Quan Zou people.Development Status and Trend of Things traffic study [J]. Application Research of Computers, 2013, 30 (8): 2256-2261.

2. Peng Yali, Zhang Zhiming, Song Yanting.Intelligent traffic based on Network Calculus Allocation [J].Application Research of Computers, 2012,29 (8): 2891-2894.

3. S. Savasta, M. Pini and G. Marfia. Performance Assessment of a Commercial GPS Receiver for Networking Applications [C], in 5th IEEE Proc. Consumer Communications and Networking Conference, 2008, 613617.

4. Wang Yan. The development of wireless personnel positioning in internet of things based on ZigBee and sensors [J]. International Journal of Digital Content Technology and its Applications, 2012, 6 (12): 47-54.

5. Ko Chien-Ho, RFID 3D location sensing algorithms [J]. Automation in Construction, 2010, 19 (5): 588-595.

6. Wang Jing, Prasad R Venkatesha, An Xueli, et al, A study on wireless sensor network based indoor positioning systems for context-aware applications [J]. Wireless Communications and Mobile Computing 2012, 12 (1): 53-70.

7. Lee Yong Up, Weighted-average based AOA parameter estimations for LRUWB wireless positioning system [J]. IEICE Transactions on Communications, 2011, E94-B (12): 3599-3602.

8. T. Manodham, L. Loyola and T. Miki, A novel wireless positioning system for seamless internet connectivity based on the WLAN infrastructure [J], Wireless Personal Communications, 2008, 44 (3): 295-309. 
9. EA Prigge and JP How, Signal architecture for a distributed magnetic local positioning system [J], IEEE Sensors Journal, 2004, 4 (6): 864-873.

10. K. Muto, K. Yagi, K. Eguchi, G. Chen, et al, Measurement result of slice positioning sound of MRI equipment [J], Acoustical Science and Technology, 2006, 27 (3):. 174-176.

\section{Fund Project}

National Natural Science Foundation of China (61202314); Gansu Province Natural Science Foundation of China (1310RJZA067); Lanzhou Jiaotong University Youth Science Foundation (2013003)

\section{About the Author}

Cao Yan (1982-), male (Han), Tai'an, lecturer, Master, research direction: the optical communication, signal processing; Leitao (1981-), male (Han), Dali, Shaanxi, associate professor, Ph.D., research interests include image processing, multimedia signal processing. 